\title{
Influência da resolução do MDE na caracterização morfométrica de bacia hidrográfica
}

\author{
Influence of the DEM resolution on the morphometric characterization of basin
}

\author{
Elias Rodrigues da Cunha \\ Discente do Programa de Pós-Graduação em Geografia pela \\ Universidade Federal da Paraíba-UFPB, Brasil \\ eliasrodriguesdacunha@hotmail.com
}

Vitor Matheus Bacani

Professor Associado da Universidade Federal de Mato Grosso do Sul

UFMS/CPTL, Brasil

vitormbacani@gmail.com

\begin{abstract}
Resumo
O objetivo do trabalho foi analisar a influência da resolução espacial do Modelo Digital de Elevação (MDE) na caracterização de bacia hidrográfica no Bioma Cerrado a partir dos modelos MDEs ALOS RTC, SRTM 30m e SRTM 90m usando SIG. O limite da bacia e a rede de drenagem foram extraídos de modo automático, enquanto os índices morfométricos linear, areal e do relevo foram calculados, são eles: densidade de drenagem, média do escoamento superficial, coeficiente de compacidade, coeficiente de manutenção, curva hipsométrica, perfil topográfico, altitude e declividade. O MDE ALOS apresentou melhor resultado na delimitação automática da bacia em relação ao MDE SRTM $30 \mathrm{~m}$ e $90 \mathrm{~m}$. Esse resultado evidência o potencial do MDE ALOS como base de dados para caracterização de bacia hidrográfica e, consequentemente, para o gerenciamento de recursos hídricos de modo rápido, preciso e sem custo, uma vez que esses dados são gratuitos. A partir da análise integrada dos índices morfométricos, concluiu-se que a bacia hidrográfica do Rio da Prata não apresenta susceptibilidade à inundação exceto em ocasiões de chuvas excepcionais que podem provocar inundação nas áreas de planícies fluviais.
\end{abstract}

Palavras-chave: Geoprocessamento, MDE ALOS, Bioma Cerrado, Rio da Prata.

\begin{abstract}
The aim of this paperwork was to analyse the influence of the spatial resolution of the Digital Elevation Model (DEM) on the characterisation of the Cerrado watershed from the ALOS RTC, SRTM 30m and SRTM 90m DEMs using GIS. The basin boundary and the drainage network were extracted automatically, while the linear, area and relief morphometric indexes were calculated: drainage density, surface runoff average, compactness coefficient, maintenance coefficient, hypsometric curve, topographic profile, height and slope. DEM ALOS showed better results in automatic watershed delimitation than DEM SRTM 30m and 90m. This result highlights the potential of DEM ALOS as a database for watershed characterisation and, consequently, for water resources management quickly, accurately and without cost, since this data is free. From the integrated analysis of the morphometric indices, it was concluded that the Prata River watershed is not susceptible to flooding except on occasions of exceptional rainfall that may cause flooding in the floodplains.
\end{abstract}

Keywords: Geoprocessing, ALOS DEM, Cerrado Biome, Prata River. 


\section{INTRODUÇÃO}

A caracterização morfométrica corresponde ao conjunto de procedimentos que caracterizam aspectos geométricos e de composição dos sistemas ambientais, servindo como indicadores relacionados à forma, arranjo estrutural, interação entre as vertentes e a rede de canais fluviais de uma bacia hidrográfica que, por sua vez, evidenciam situações e valores que extrapolam as questões hidrológicas e geomorfológicas (HORTON, 1945; STRAHLER, 1958; SCHUMM, 1956; CHTRITOFOLLETI, 1970; VILLELA; MATTOS, 1975). Neste sentido, as características físicas de uma bacia são elementos de grande importância em seu comportamento hidrológico.

Em estudos hidrológicos, é essencial a análise das características físicas de bacias hidrográficas. As características físicas e bióticas de uma bacia exercem importante papel nos processos do ciclo hidrológico influenciando em diversos fatores e processos como, a infiltração, a quantidade de água produzida como deflúvio, evapotranspiração e o escoamento superficial e subsuperficial (TONELLO et al., 2006).

O uso de Modelo Digital de Elevação (MDE) por meio Sistema de Informação Geográfica (SIG) permite métodos para analisar características hidrológicas e topográficas com vantagens operacionais e qualidade. Os MDEs apresentam-se como uma maneira de obtenção de informação veloz e precisa. Assim, o avanço do Sensoriamento Remoto (SR) e novas técnicas de geoprocessamento automatizaram esses estudos (BERA et al., 2014; NIKOLAKOPOULOS et al., 2015; DAS et al., 2016), que antes demandavam dispendiosos e longos levantamentos de campo e gabinete.

MDE refere-se a um modelo quantitativo da superfície terrestre através de uma representação numérica da elevação do terreno em formato digital (FELICÍSIMO, 1994). Um MDE pode ser definido por meio de i) contornos com pares de coordenadas x, y ao longo de cada linha de contorno de uma elevação especificada, ii) uma rede irregular triangulada constituída por nós e linhas irregularmente distribuídos com coordenadas tridimensionais ( $x, y$ e $z$ ) e iii) uma matriz bidimensional de números que representa a distribuição espacial das elevações em uma grade regular (VILLAVERDE et al., 2015).

A crescente disponibilidade gratuita de MDE a partir do ano 2000 com as missões SRTM (Shuttle Radar Topographic Mission), ASTER (Advanced Spaceborne Thermal Emission and Reflection Radiometer) ALOS PALSAR (Advanced Land Observing Satellite/ Phase Array type Lband Synthetic Aperture Radar) promoveram um ganho enorme para a comunidade cientifica, devido a possibilidade de realizar estudos em escala de maior detalhe, precisão de maneira gratuita.

Com a liberação do MDE SRTM 3 arc 90m para a América do Sul (2003), ocorreu uma expectativa com relação aos ganhos de conhecimento sobre nosso território, justificada pela carência 
geral de dados topográficos em escalas adequadas. No entanto, esse MDE contém imperfeições caracterizadas por depressões espúrias, picos anômalos, pontos ou áreas com ausência de dados, além de apresentar uma baixa resolução espacial. Na busca de corrigir essas falhas, a Embrapa Monitoramento por Satélite desenvolveu, em 2005, o projeto Brasil em Relevo com o objetivo de construir uma base nacional de dados topográficos, colocando à disposição um MDE de melhor qualidade para todo o território nacional.

Sendo que, somente a partir de setembro de 2014 foram disponibilizados MDE SRTM 1 arc $\sim 30 \mathrm{~m}$ com uma média resolução espacial para a América do Sul. Em outubro do mesmo ano, foi liberado o MDE ALOS/RTC (Radiometric Terrain Corrected) de alta resolução espacial $\sim 12,5 \mathrm{~m}$ pela Alaska Satellite Facility. Esses dados incluídos no projeto RTC são feixes finos e cenas polarimétricas em todas as áreas terrestres globais, exceto na Antártica, na Groenlândia, na Islândia e no norte da Eurásia.

Nas últimas décadas, foram realizados muitos estudos utilizando MDEs para comparar e analisar a modelagem das características da hidrologia superficial, a análise morfométrica (PATEL et al., 2013; SREEDEVI et al., 2013; VIECELI et al., 2015), a avaliação de rede de drenagem (PAUL et al., 2015; MOKARRAM et al., 2017; SOUSA e PAZ, 2017), e para a identificação de áreas de inundação (PAN et al., 2013; HSU et al., 2016). Dentro do contexto, o presente trabalho objetivou analisar a influência da resolução espacial do MDE na caracterização de uma bacia hidrográfica no Bioma Cerrado.

\section{METODOLOGIA}

\section{1 Área de estudo}

A bacia hidrográfica do Rio da Prata compreende uma área de $1392 \mathrm{~km}^{2}$. É localizada entre os municípios de Bonito, Jardim e Porto Murtinho no sudoeste do Estado de Mato Grosso do Sul, entre as latitudes de $21^{\circ} 16^{\prime} 12^{\prime \prime}$ e $21^{\circ} 39^{\prime} 36^{\prime \prime}$ S e longitudes de 56 $12^{\prime} 36^{\prime \prime}$ e 56 46 48" W (Figura 1).

O Rio da Prata destaca-se do ponto de vista fisiográfico por se apresentar entre o Planalto da Bodoquena e Depressão Pantaneira. Apesar de encontrar-se no Bioma Cerrado, sofre influência da transição dos biomas: Mata Atlântica e Cerrado nas adjacências do Pantanal Sul-Mato-Grossense. Estes aspectos lhe conferem peculiaridades ambientais riquíssimas ligadas à fauna, flora e, sobretudo geológica-geomorfológicas.

A geologia é constituída por mármores do Grupo Cuiabá; diamictito, arenito, folhelho e siltito da Formação Aquidauana; arenitos da Formação Botucatu; calcários calcítico e dolomítico da Formação Cerradinho; tufa da Formação Xaraiés (SALLUN FILHO, 2009); calcário dolomítico da Formação Bocaina (CPRM, 2006). 


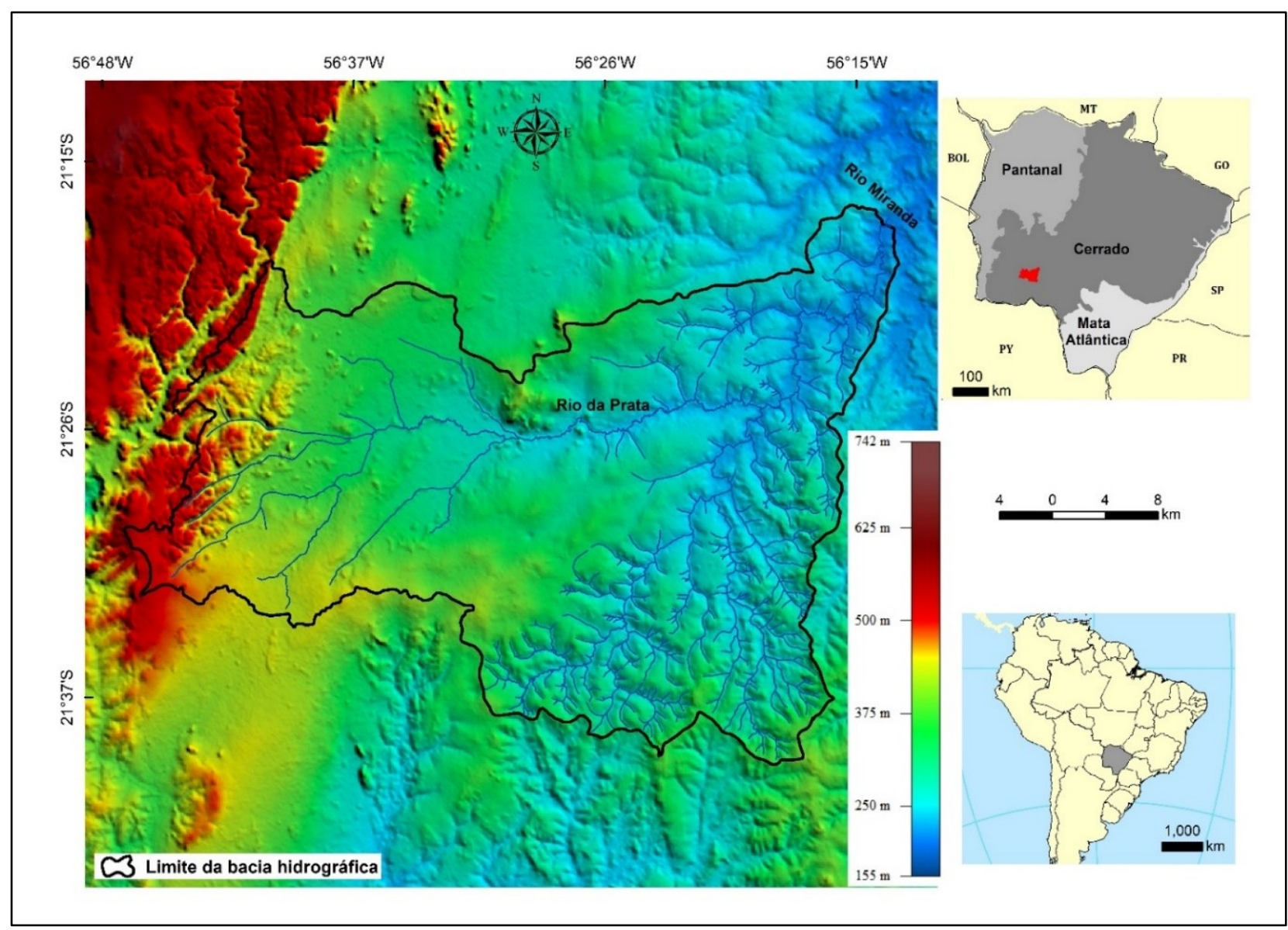

Figura 1- Localização da área de estudo sobre o MDE SRTM 30m.

Fonte: Organização dos autores.

O clima da região é classificado como úmido, apresenta índice efetivo de umidade com valores anuais variando de 40 a $60 \%$. A precipitação pluviométrica anual é a mais elevada do Estado de Mato Grosso do Sul, variando entre 1.750 a $2.000 \mathrm{~mm}$ anuais. O excedente hídrico anual é de 1.200 a $1.400 \mathrm{~mm}$ durante um período de sete a oito meses e a deficiência hídrica é registrada entre 200 e $350 \mathrm{~mm}$ durante três meses. A temperatura média anual é $22^{\circ} \mathrm{C}$, destacando-se como a mais baixa do Estado (MS, 1990).

\subsection{Processamento MDE/RTC ALOS, SRTM 30m e SRTM 90m}

Para a correção dessas pequenas depressões, foi aplicada uma técnica em todos os MDEs utilizando a, função pit removal disponível no Terrset 18.31 que leva em consideração a altitude da célula vizinha para preencher essas falhas. As falhas no MDE denominadas pit ou sinks, são caracterizadas por áreas rodeadas de elevações com valores de cotas superior, semelhante a uma depressão. O preenchimento dessas pequenas depressões é o primeiro tratamento dado à matriz de altitudes. As depressões podem ser componentes reais do terreno, mas também podem ser oriundas de erros de digitalização, artefatos produzidos na interpolação dos MDE ou processo de 
reamostragem (WU et al., 2008). Eles causam obstáculos no cálculo de direção de fluxo, levando a gerações imprecisas no fluxo acumulado.

O MDE ALOS com resolução espacial de $12.5 \mathrm{~m}$ foi obtido gratuitamente do Alaska Satellite Facility, já corridos nos processos radiométrico e terreno. Sendo que, a correção radiométrica envolve a remoção de influências enganosas da topografia sobre os valores de retroespalhamento. No caso da correção de terreno, a mesma objetiva corrigir as distorções geométricas que levam a erros de geolocalização. Small (2011) analisou de forma abrangente as várias técnicas de correção radiométrica do terreno e concluiu que a abordagem de integração de área de pixel é a técnica mais robusta disponível para normalizar a imagem SAR. Para o recobrimento da área de estudo foi necessária a elaboração de um mosaico, através da ferramenta Mosaic (disponível no Terrset 18.31) quadrículas Ap_17402_Plr_F6750_Rt1, Ap_17402_Plr_F6760_Rt1, Ap_27467_Plr_F6760_Rt1, Ap_27890_Plr_F6760_Rt1 formato Geotiff (16 bits) nas coordenadas UTM zona 21s.

O MDE SRTM 3 arc $\sim 90$ m quadricula sf-21-X-c no formato Geotiff (16 bits), coordenadas Lat/Long foi obtido no site da Brasil Relevo. Os dados brutos de elevação passaram por correção e padronização, eliminando falhas, sombras e distorções (MIRANDA et al., 2005). O preenchimento dos pontos ou áreas sem informação no modelo foi realizado utilizando-se como fonte de informação adicional os MDEs disponibilizados pela FAO/UNEP, no âmbito da rede de cooperação Global Land Cover Network (GLCN), corrigidos com a utilização do aplicativo ArcInfo. A partir do sitio Earth Explorer (https://earthexplorer.usgs.gov/) foi realizado o download do MDE SRTM 1 arc $\sim 30 \mathrm{~m}$ quadrícula s22_w057_1arc_v3 (formato Geotiff, 16 bits).

\subsection{Limite da bacia e rede de drenagem (referência e automática)}

A delimitação da bacia e extração da rede de drenagem (referência) foram feitas a partir da análise e interpretação do MDE (extração de curvas de 12,5 m), mosaico de cartas topográficas (folha Boqueirão, Jardim, Rio Perdido e Fazenda Margarida) escala 1:100.000, imagem de satélite Landsat 8 sensor OLI, órbita/ponto 226/75 datada de 18/03/2016 e imagem de alta resolução disponível no Arcgis online.

Para a extração automática da rede de drenagem e bacia hidrográfica a partir dos MDEs foram percorridas as seguintes etapas: correções das depressões (descrito acima), direção de fluxo (flow direction), fluxo acumulado (flow accumulation) e extração da rede de drenagem e bacia hidrográfica. A direção de fluxo foi calculada utilizando algoritmo 8D. Assim, para cada pixel foram examinados os oito pixels vizinhos e escolhida direção da inclinação mais íngreme (Jensen e DOMINGUE, 1988). Para gerar o fluxo acumulado foi calculado para cada pixel do MDE o número de pixels à montante cujos caminhos fluem para essa célula. Os maiores valores de acumulação foram 
usados para definir o exutório da bacia. A partir dos fluxos acumulados nas células foi extraída a rede de drenagem considerando limiares das áreas de drenagens de $1 \mathrm{~km}^{2}, 2 \mathrm{~km}^{2}, 5 \mathrm{~km}^{2}$ e $10 \mathrm{~km}^{2}$. A seguir, o fluxograma (Figura 2) ilustra as etapas e métodos envolvidos na extração automática da rede de drenagem, limite da bacia e outros parâmetros do Rio da Prata, utilizando MDE em ambiente Terrset 18.31 .

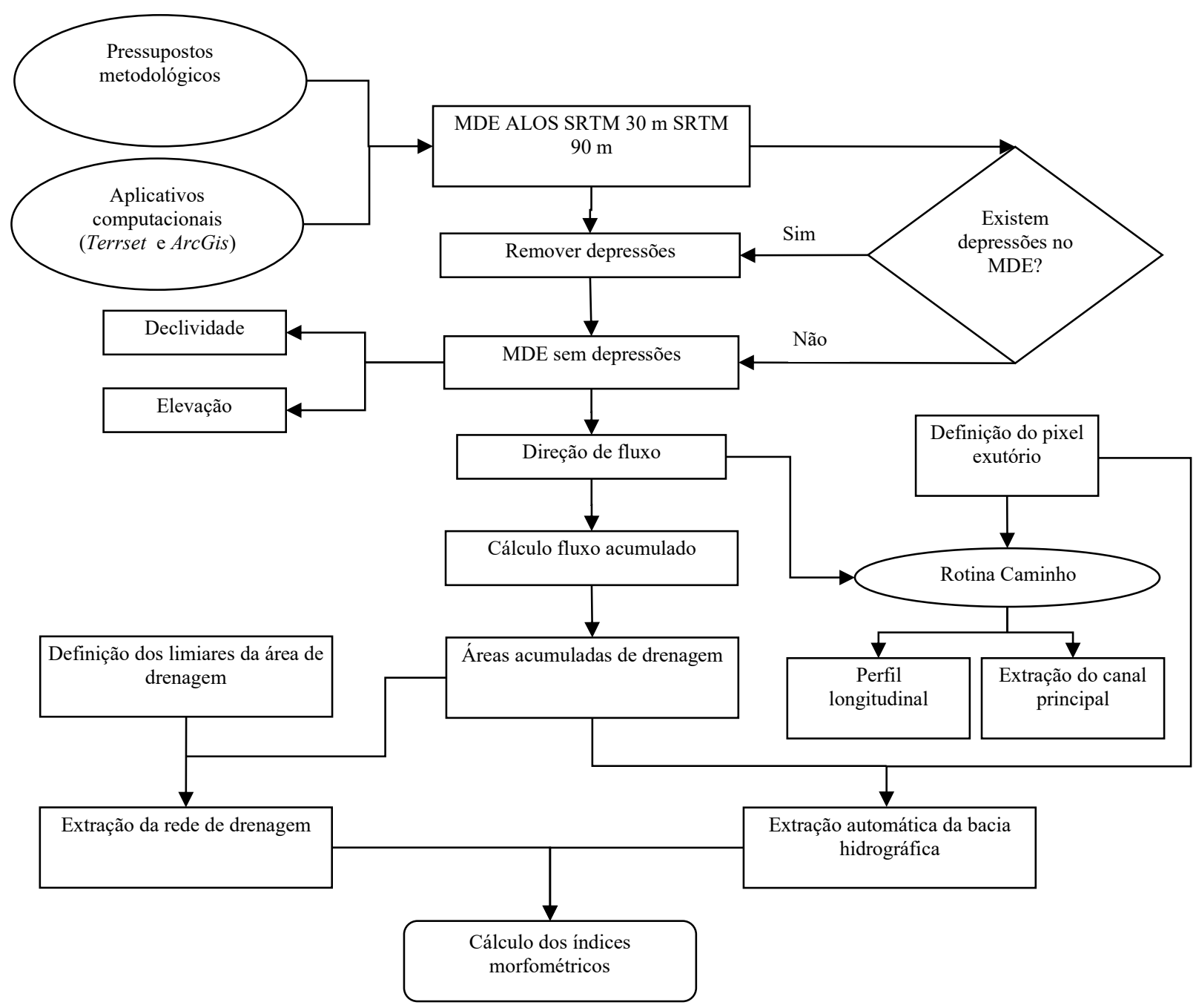

Figura 2- Fluxograma das etapas para a extração do limite da bacia, rede de drenagem e cálculo dos índices morfométricos a partir dos diferentes MDEs.

Os índices morfométricos areal, linear e relevo (Quadro 1) foram obtidos a partir das ferramentas Gis analysis (Terreset 18.31) e Spatial analysis softwares (ArcMap 10). Outros índices da bacia foram calculados através das equações (descritas abaixo) no software Excel ${ }^{\circledR}$, bem como a elaboração dos quadros e figuras. 
Quadro 1- Índices morfométricos calculados.

\begin{tabular}{|c|c|c|c|}
\hline Parâmetros & Fórmula/ Relação & Descrição & Referências \\
\hline $\begin{array}{l}\text { Lr- Comprimento } \\
\text { do rio principal }\end{array}$ & $\begin{array}{l}\text { Canal de ordem mais } \\
\text { elevada }\end{array}$ & & Horton (1945) \\
\hline $\begin{array}{l}\text { Lt- Comprimento } \\
\text { total de todos os } \\
\text { canais }(\mathrm{km})\end{array}$ & $\begin{array}{l}\text { Soma de todos os } \\
\text { canais da bacia }\end{array}$ & & Horton (1945) \\
\hline $\begin{array}{c}\text { A- Área de } \\
\text { drenagem }\left(\mathrm{km}^{2}\right)\end{array}$ & & $\begin{array}{l}\text { É toda área drenada pelo conjunto do } \\
\text { sistema fluvial. }\end{array}$ & $\begin{array}{l}\text { Christofoletti } \\
\text { (1980) }\end{array}$ \\
\hline P- Perímetro (km) & & Medida do contorno bidimensional da bacia & $\begin{array}{l}\text { Christofoletti } \\
\text { (1980) }\end{array}$ \\
\hline $\begin{array}{l}\text { Extensão média do } \\
\text { escoamento } \\
\text { superficial }\end{array}$ & $l=A / 4 L^{\prime}$ & $\begin{array}{l}\text { Onde: } l=\text { extensão média do escoamento } \\
\text { superficial, } \mathrm{A}=\text { área da bacia e } L^{\prime}= \\
\text { comprimento total dos cursos d'água. }\end{array}$ & $\begin{array}{l}\text { Villela e Matos, } \\
1975\end{array}$ \\
\hline $\begin{array}{l}\text { Kc- Coeficiente de } \\
\text { compacidade }\end{array}$ & $K c=0,28 \frac{P}{\sqrt{A}}$ & $\begin{array}{c}\text { Onde: } \mathrm{Kc}=\text { coeficiente de compacidade, } \mathrm{P}= \\
\text { perímetro e } \mathrm{A}=\text { área da bacia. }\end{array}$ & Horton (1945) \\
\hline $\begin{array}{l}\text { Tc-Taxa de } \\
\text { circulação }\end{array}$ & $\mathrm{Tc}=4 \pi * \mathrm{~A} / \mathrm{P}^{2}$ & $\begin{array}{c}\text { Onde: } \mathrm{A}=\text { área da bacia, } \mathrm{P}=\text { Perímetro da } \\
\text { bacia. }\end{array}$ & Strahler (1964) \\
\hline $\begin{array}{l}\text { D } d \text { - Densidade de } \\
\text { drenagem }\left(\mathrm{km} / \mathrm{km}^{2}\right)\end{array}$ & $\mathrm{D} d=\frac{L_{t}}{A}$ & $\begin{array}{c}\text { Onde: } D d=\text { densidade de drenagem, } \mathrm{L}_{\mathrm{t}}= \\
\text { comprimento total dos cursos d'água, } \mathrm{A}= \\
\text { área da bacia }\end{array}$ & Horton (1945) \\
\hline $\begin{array}{l}\text { Cm- Coeficiente de } \\
\text { manutenção }\end{array}$ & $\mathrm{Cm}=\frac{1}{D d} \cdot 1000$ & $\begin{array}{c}\text { Onde: } \mathrm{Cm}=\text { Coeficiente de manutenção, } \\
\mathrm{Dd}=\text { densidade de drenagem } \\
\text { canal de escoamento. }\end{array}$ & Schumm (1956) \\
\hline $\begin{array}{l}\text { Ir- Índice de } \\
\text { Rugosidade }\end{array}$ & $\mathrm{H} * \mathrm{Dd}$ & $\begin{array}{l}\text { Onde: } \mathrm{H}=\text { amplitude altimétrica, } \\
\mathrm{Dd}=\text { densidade de drenagem }\end{array}$ & Schumm (1956) \\
\hline Curva hipsométrica & & $\begin{array}{l}\text { É a representação gráfica do relevo médio } \\
\text { de uma bacia. }\end{array}$ & Schumm (1956) \\
\hline Declividade & & $\begin{array}{c}\text { A declividade é o ângulo de inclinação da } \\
\text { superfície local em relação ao plano } \\
\text { horizontal. }\end{array}$ & Ross (1994) \\
\hline
\end{tabular}

\section{RESULTADOS E DISCUSSÃO}

As propriedades morfométricas linear, areal e relevo da bacia hidrográfica do Rio da Prata, extraídas dos diferentes MDEs e calculadas são apresentadas no Quadro 2, bem como os parâmetros da bacia de referência. Com base nesses resultados foi constatado que a resolução espacial dos MDEs influenciou diretamente nos valores dos parâmetros.

A partir uma análise integrada e a média dos resultados obtidos dos MDEs para o coeficiente de compacidade Kc $(2,40)$, Taxa de circulação Tc $(0,16)$ e densidade de drenagem Dd $(0,60)$ foi possível verificar a baixa susceptibilidade da bacia a enchente. Uma vez que a bacia apresenta formato alongado, extenso e uma média densidade de drenagem, favorecendo o escoamento superficial das águas pluviais. Outro índice que valida a susceptibilidade da bacia a enchentes é a relação entre a extensão média do escoamento superficial (L) e a distância média a ser percorrida pela água precitada. Esta distância é calculada por uma linha reta que sai do ponto onde ocorreu a sua precipitação até o ponto mais próximo do leito de qualquer curso d'água (VILLELA; MATTOS 1975). Neste estudo foi encontrado uma distância média de $420 \mathrm{~m}$, ao comparar com os valores de 114 m encontrados por 
OLSZEVSKI et al., (2011) pode-se considerar um valor elevado, ou seja, com baixa propensão à alagamentos em caso de chuvas torrenciais, exceto nas áreas de planície de inundação.

Quadro 2- Índices morfométricos calculados a partir dos MDEs.

\begin{tabular}{|c|c|c|c|c|c|}
\hline Índices morfométricos & ALOS & SRTM 30 m & SRTM 90 m & Referência & Variação \% \\
\hline Lr- Comprimento do rio principal & 102,91 & 104,10 & 90,59 & 98,93 & 6,01 \\
\hline A- Área de drenagem $\left(\mathrm{km}^{2}\right)$ & 1402,01 & 1395,69 & 1362,08 & 1392,96 & 1,04 \\
\hline A- Área de drenagem omitida $\left(\mathrm{km}^{2}\right)$ & 15,11 & 16,61 & 51,80 & & 154 \\
\hline A-Área de drenagem incluída $\left(\mathrm{km}^{2}\right)$ & 24,16 & 19,36 & 21,23 & & 11,30 \\
\hline Distância média das bacias $(\mathrm{km})$ & 0,17 & 0,16 & 0,32 & & 92,16 \\
\hline P- Perímetro (km) & 379,10 & 372,56 & 295,71 & 230,33 & 33,18 \\
\hline Dd- Densidade de drenagem $\left(\mathrm{km} / \mathrm{km}^{2}\right)$ & 0,63 & 0,62 & 0,54 & 0,57 & 7,71 \\
\hline Lt- Comprimento de todos os canais $(\mathrm{km})$ & 884,91 & 863,98 & 732,70 & 794,43 & 8,90 \\
\hline L- Extensão escoamento superficial (m) & 400 & 400 & 460 & 440 & 8,12 \\
\hline Cm-Coeficiente de manutenção & 1,51 & 1,61 & 1,91 & 1,76 & 11,24 \\
\hline Ir- Índice de rugosidade & 0,25 & 0,24 & 0,20 & 0,22 & 10,11 \\
\hline Tc- Taxa de circulação & 0,12 & 0,12 & 0,19 & 0,33 & 132,85 \\
\hline Kc- Coeficiente de compacidade & 2,83 & 2,79 & 2,24 & 1,73 & 33,21 \\
\hline Elevação máxima (m) & 609 & 595 & 584 & & 2,75 \\
\hline Amplitude altimétrica (m) & 392 & 388 & 377 & & 2,56 \\
\hline Altitude média (m) & 338,22 & 327,10 & 323,50 & & 2,91 \\
\hline Altitude mínima (m) & 217 & 207 & 207 & & 3,07 \\
\hline Declividade média \% & 5,81 & 4,87 & 2,25 & & 43,75 \\
\hline
\end{tabular}

*A rede de drenagem utilizada como base foi extraída a partir MDE ALOS com limiar de área igual a $2 \mathrm{~km}^{2}$ por apresentar melhor resultado em relação aos outros limiares.

No caso do comprimento do rio principal, verificou-se que o canal de maior fluxo 104,10 km (SRTM 30 m) e 102,91 km (ALOS) foi extraído dos MDEs de média e alta resolução espacial. Embora o canal extraído do MDE SRTM 90 m tenha apresentado uma diferença de 8,34 km em relação ao canal de referência, no geral, ocorreu uma variação de apenas 6,01\% nos valores encontrados para esse parâmetro.

Ao analisar as áreas das bacias derivadas da extração automática (Figura 3) com a bacia de extraída manualmente, observou-se que os MDEs ALOS e SRTM 30m omitiram apenas 1,08 km² e $1,19 \mathrm{~km}^{2}$ respectivamente. Os valores baixos demonstram que esses MDEs são compatíveis com a delimitação manual, apresentando-se como uma base de dados confiável para estudos hidrológicos. No entanto, houve uma omissão extremamente alta $\left(51,90 \mathrm{~km}^{2}\right)$ na área de drenagem extraída do MDE SRTM 90 m, valor três vezes maior do que os obtidos nos outros MDEs, com variação de 158 \% entre o resultado: Quando comparadas as áreas incluídas, a variação apresentada é de apenas $11,30 \%$ o que demostra valores pouco discrepantes. 
a) ALOS $12.5 \mathrm{~m}$

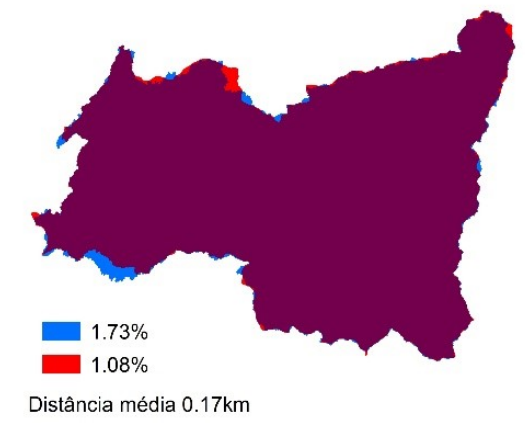

b) SRTM $30 \mathrm{~m}$

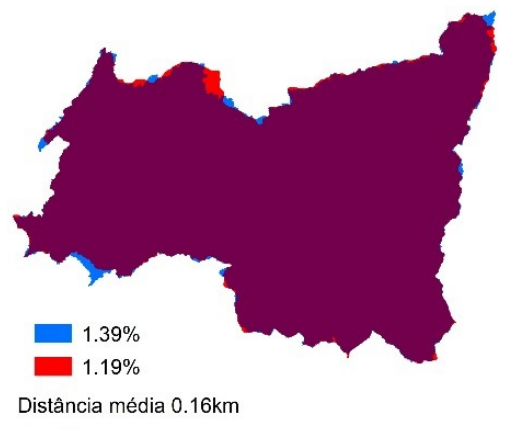

Erro de inclusão

Erro de omissão Área em acordo

Figura 3 - Comparação entre as bacias extraídas dos MDEs de modo automático com a bacia de referência.

A análise visual da rede de drenagem extraída dos MDEs com os diferentes limiares $1 \mathrm{~km}^{2}$, $2 \mathrm{~km}^{2}, 5 \mathrm{~km}^{2}$ e $10 \mathrm{~km}^{2}$ mostra que o limiar de área $2 \mathrm{~km}^{2}$ obtido do MDE ALOS apresentou melhor resultado quando comparado à drenagem de referência. Sendo que o comprimento total dos canais obtidos do MDE ALOS apresentou uma diferença 90,48 km e MDE SRTM $30 \mathrm{~m}$ um valor de $69,55 \mathrm{~km}$, tal diferença se dá ao maior detalhamento da drenagem nas áreas de banhado extraídas do MDE ALOS.

Segundo Horton (1945) a densidade de drenagem (Dd) depende do comportamento hidrológico das rochas. Assim, nas rochas mais impermeáveis, as condições para o escoamento superficial são melhores possibilitando a formação de canais e, consequentemente, aumentando a densidade de drenagem. O contrário acontece com rochas de granulometria grossa. A unidade de estudo apresenta uma Dd regular com valor médio de $0,60 \mathrm{~km} / \mathrm{km}^{2}$ e variação máxima de 9,52\%. Essa diferença $(90,48 \mathrm{~km})$ é explicada porque o comprimento total dos canais derivados do MDE ALOS é maior do que a drenagem de referência.

A partir da extração da rede drenagem automática e manual (Figura 4), foi possível verificar que a bacia apresenta dois padrões de drenagens: o paralelo (região SW e NW) e o padrão dendrítico. O primeiro é influenciado pela geologia e registra a ocorrência de afloramento, falhamento e lineamento de calcário das formações Cerradinho e Xaraiés (CPRM, 2006). O padrão dendrítico tem forte controle estrutural onde localiza a maior densidade de canais. Essa maior ramificação é reflexo da estruturação da rede de drenagem que ocorre sobre os arenitos da Aquidauana (CPRM, 2006), que apresenta rochas de resistência uniforme e estruturas sedimentares. 


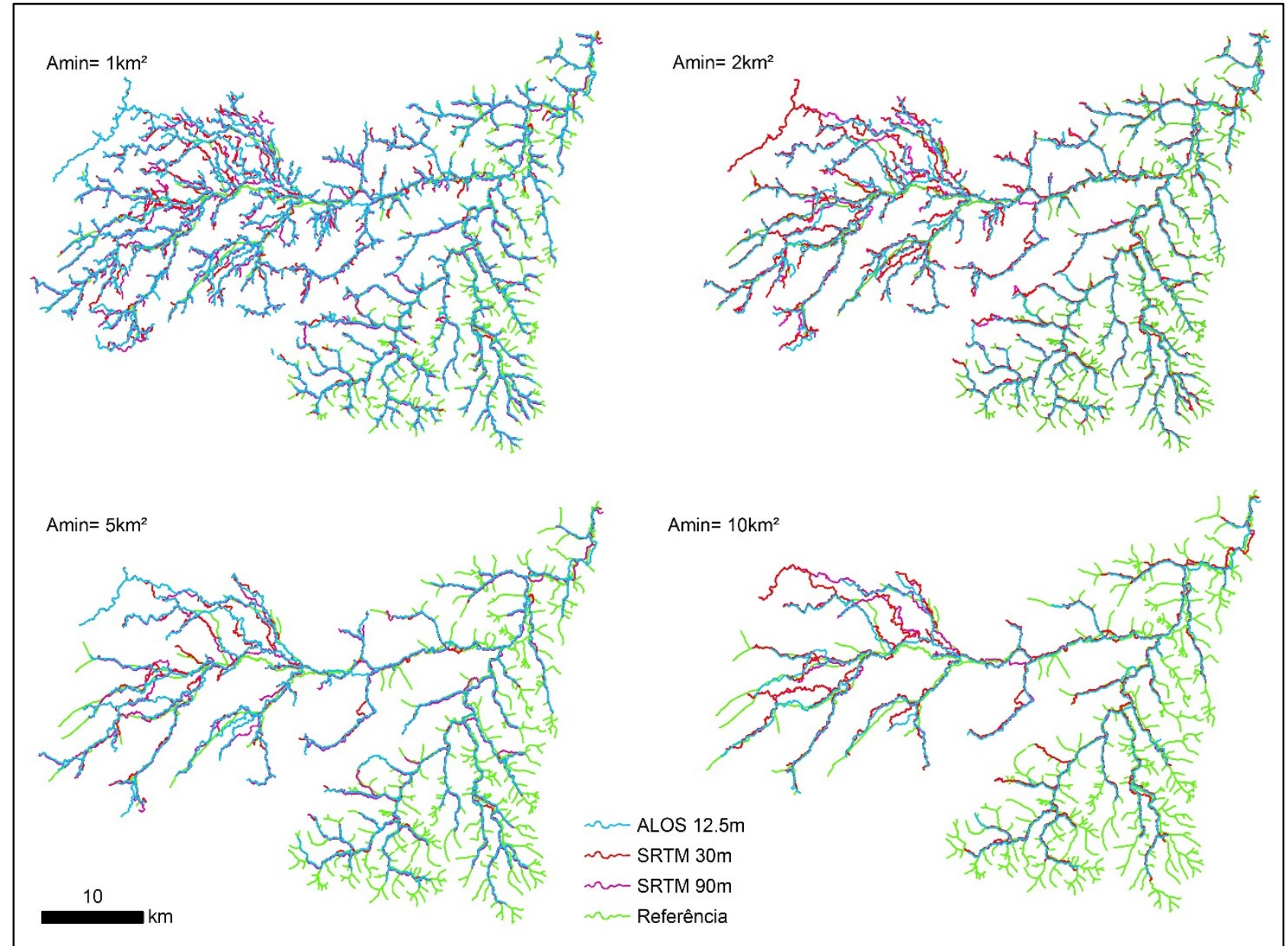

Figura 4- Rede de drenagem extraídas de modo automático a partir dos MDEs com diferentes limiares de área $\left(1 \mathrm{~km}^{2}\right.$, $2 \mathrm{~km}^{2}, 5 \mathrm{~km}^{2}$ e $10 \mathrm{~km}^{2}$ ) com a rede de drenagem de referência.

Considerando que as características do relevo podem influenciar na quantidade de radiação que a bacia hidrográfica recebe e, consequentemente, na evapotranspiração, na temperatura e na precipitação (TONELLO et al., 2006; SANTOS et al., 2012), fica evidente que a proximidade da bacia com a Serra da Bodoquena influência nos valores elevados de precipitação (1750 a 2000 $\mathrm{mm} / \mathrm{ano}$ ), considerados os mais altos/maiores de Mato Grosso do Sul.

Os valores de elevação variaram entre $207 \mathrm{~m}$ a $609 \mathrm{~m}$ para os MDEs, a cota mínima está localizada na confluência do Rio da Prata e Miranda (nordeste) e a máxima na borda da Serra da Bodoquena (leste), configurando uma amplitude de $385,67 \mathrm{~m}$. Constatou-se que o elevado valor da amplitude favorece o escoamento superficial difuso. A altitude média encontrada foi 329,61 m, com base nos resultados de elevação a partir dos três MDEs foi elaborada uma representação gráfica da curva hipsométrica (Figura 5). A análise do gráfico revela que $70 \%$ da bacia apresenta elevações inferiores a $380 \mathrm{~m}$. 


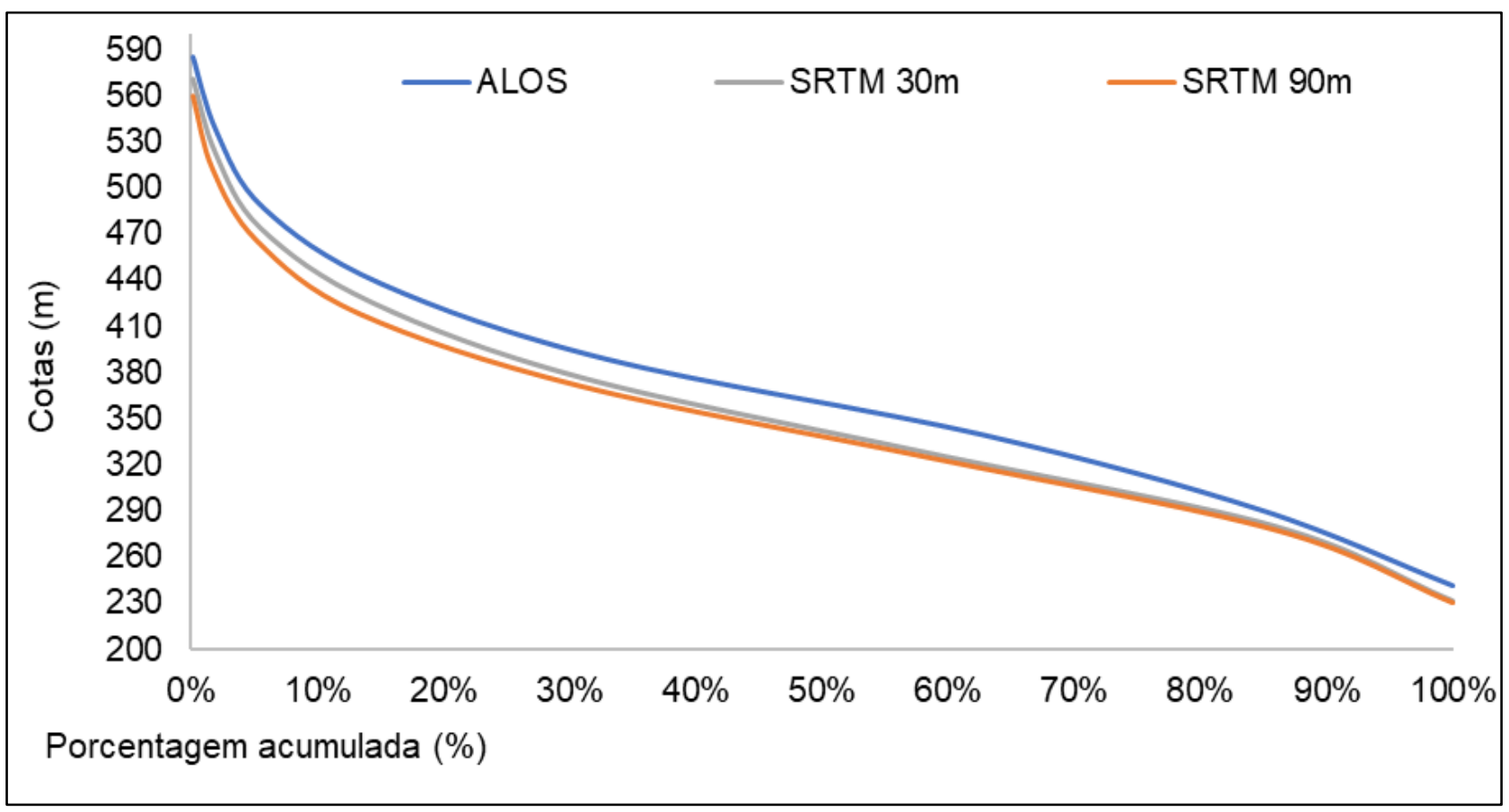

Figura 5- Curva hipsométrica extraída dos MDEs ALOS, SRTM 30m e SRTM 90m.

A partir da correlação das cotas de elevação (m) e extensão do canal principal, foi possível gerar o perfil longitudinal para os três MDEs (Figura 6). Uma maneira simples e eficaz de se determinar os pontos ou trechos anômalos (em desequilíbrio) nos perfis longitudinais de cursos d'água é a plotagem da curva de melhor ajuste. A comparação de tal curva com o perfil longitudinal mostrará os principais afastamentos, tanto positivos (trechos que se encontram acima da linha de melhor ajuste), quanto negativos (trechos situados abaixo da linha de melhor ajuste) (GUEDES et al., 2006).

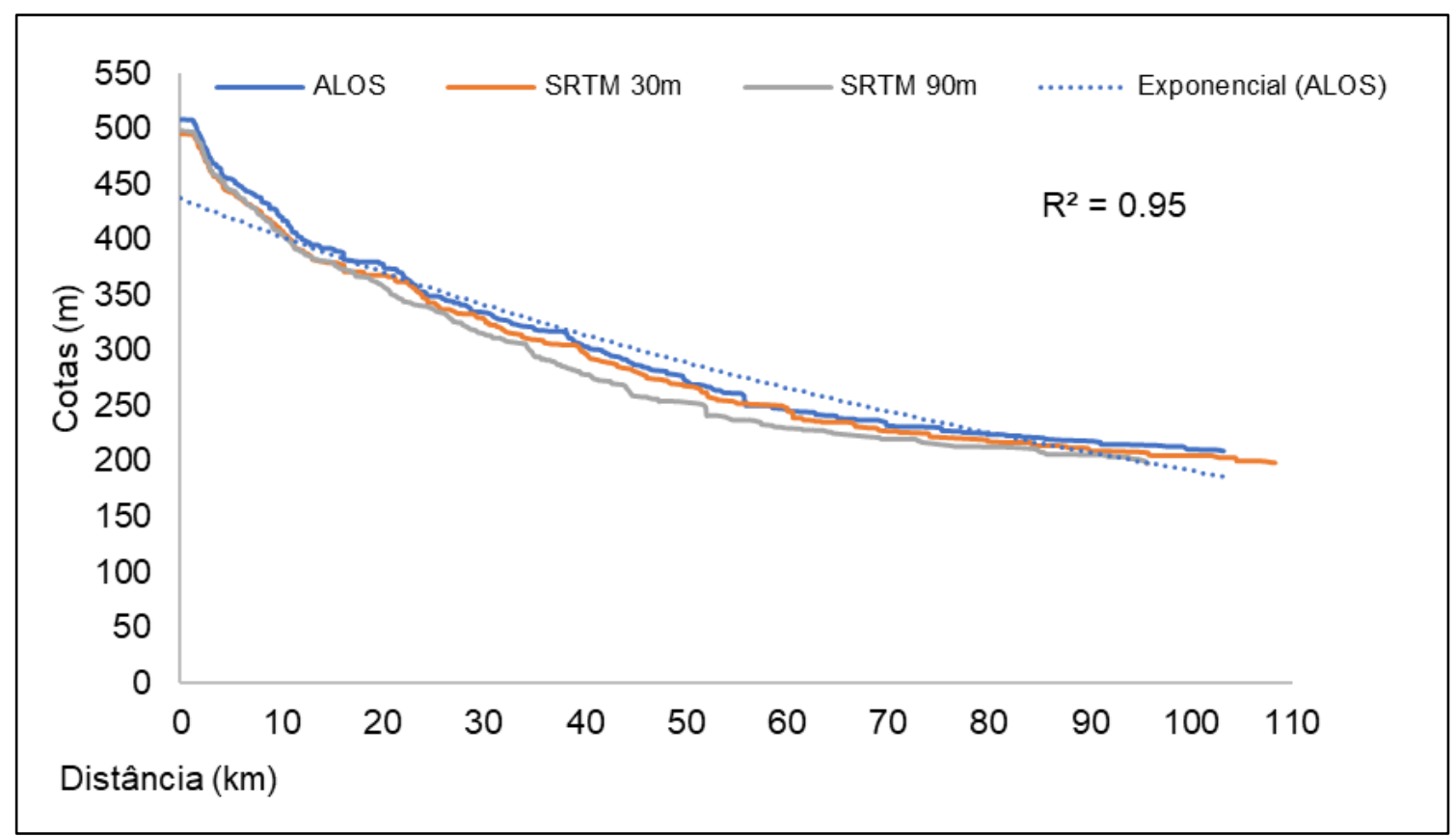

Figura 6- Perfis longitudinais com base nos MDEs ALOS, SRTM 30m e SRTM 90m. 
Ao observar o perfil longitudinal, foi possível verificar uma anomalia negativa (entre o km 10 e km 40) na área onde se localiza o Banhado do Rio da Prata. Essa região se estrutura sobre a Formação Xaraiés, caracterizada por depósitos de tufas calcárias porosas e cavernosas com os espaços vazios contendo calcita recristalizada. (CPRM, 2006). Corroborando com nossos resultados, Acklas Jr. et al. (2003) relacionam essa anomalia com resistência à erosão do substrato rochoso.

A declividade é uma importante característica de análise de uma bacia hidrográfica. Ela está relacionada com a velocidade em que se dá o escoamento superficial, o que afeta o tempo que a água da chuva leva para se concentrar nos leitos fluviais que constituem a rede de drenagem das bacias, sendo que os picos de enchente, infiltração e susceptibilidade para erosão dos solos, dependem da rapidez com que ocorre o escoamento sobre o terreno da bacia (VILLELA; MATTOS, 1975). Assim, é essencial a compreensão da distribuição da inclinação do relevo, uma vez que ela fornece informações para o planejamento e mecanização da agricultura, planejamento de estruturas de engenharia, práticas conservacionistas entre outros (SREEDEVI et al., 2009).

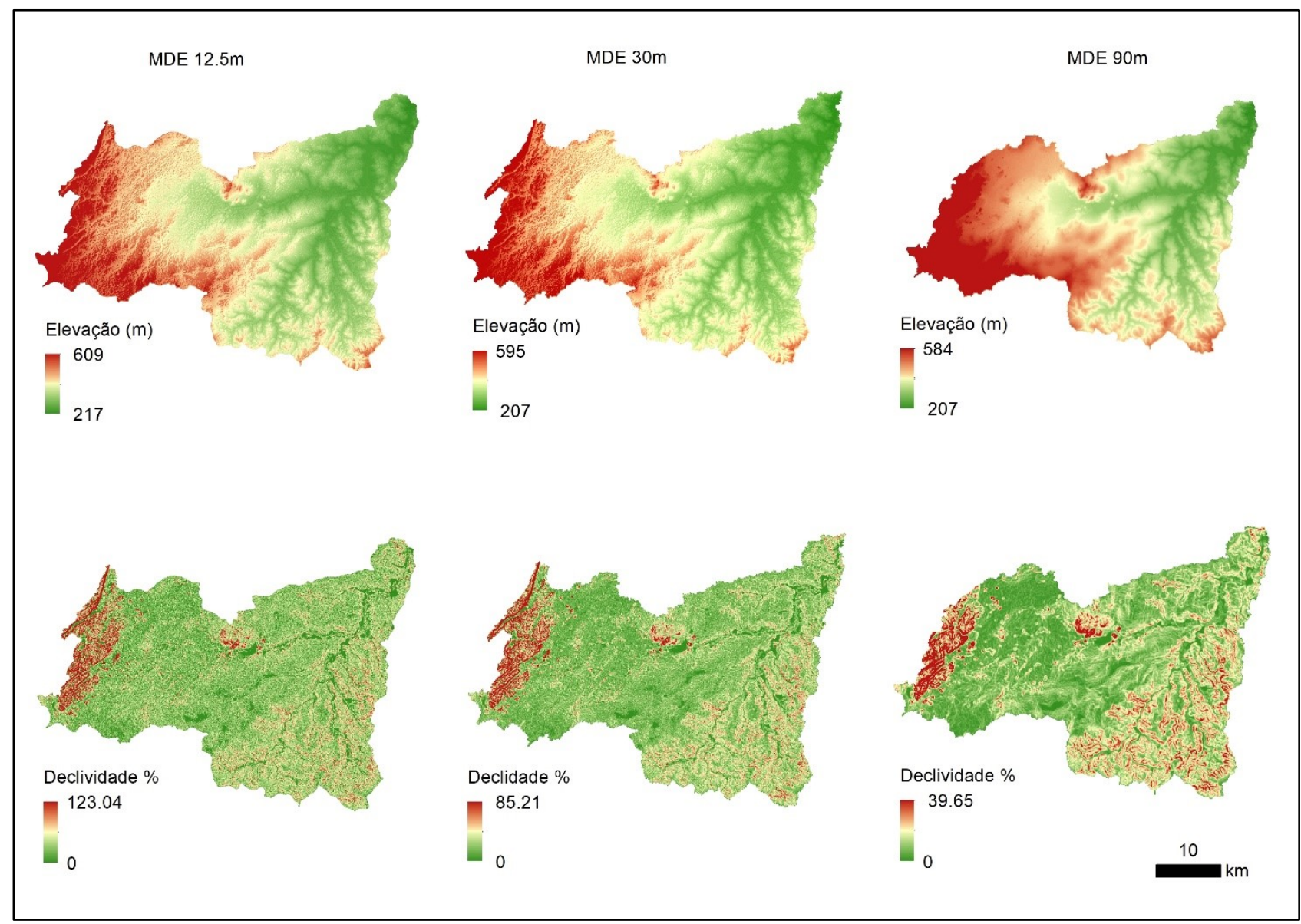

Figura 7- Distribuição da elevação e declividade da bacia hidrográfica do Rio da Prata.

Ao comparar os resultados de declividade (Figura 7) fica evidente o melhor detalhamento nos resultados MDE ALOS, apresentando variação de 0 a 123\%, enquanto o MDE SRTM 90 m teve inclinações entre 0 a 39.65\%. Ao analisar a declividade média entres as bases ocorreu uma variação 
de $61.27 \%$. De modo geral, na bacia ocorre o predomínio de relevo suave ondulado (colinas), sendo que as áreas com maiores declividades (superiores a 45 \%) ocupação o leste da bacia, em sua maioria localizadas na Serra da Bodoquena.

\section{CONCLUSÕES}

1) O MDE ALOS apresentou melhor resultado na delimitação automática da bacia em relação ao MDE SRTM 30 m e 90 m, omitindo apenas 1,09 km² de área;

2) Esse resultado evidencia o potencial do MDE ALOS como base de dados para caracterização de bacias hidrográficas, e consequentemente gerenciamento de recursos hídricos de modo rápido, preciso e sem custo, uma vez que esses dados são gratuitos;

3) A extração rede de drenagem pelo método $8 \mathrm{D}$ é uma alternativa eficiente e rápida nas áreas de banhado (relevo plano), considerando que a delimitação manual nessas áreas é comprometida pela dificuldade de obtenção de curvas de nível e interpretação visual de imagens de satélite de média e alta resolução;

4) Elevações inferiores a 380m ocupam $70 \%$ da bacia;

5) As inclinações superiores a $45 \%$ ocorrem nas áreas de cabeceiras (Serra da Bodoquena), regiões de relevo íngreme apresentam escoamento superficial mais rápido e volumoso favorecendo o desenvolvimento de processos erosivos.

6) A bacia hidrográfica do Rio da Prata não apresenta susceptibilidade à enchente, embora possa ocorrer inundações em áreas de planície fluvial em caso de chuvas excepcionais.

\section{AGRADECIMENTOS}

À Universidade Federal de Mato Grosso do Sul e Universidade Federal da Paraíba. A Alaska Satellite Facility pela disponibilidade do MDE ALOS. Ao United States Geological Survey (USGS) pelo MDE SRTM. À Cláudia Camargo pela correção do texto. E por fim, ao Msc. Alex Balbuena, coordenador do International TEFL Trainning Institute (ITTI-MS) pela tradução do abstract.

\section{REFERÊNCIAS}

ACKLAS JR. R.; ETCHEBEHERE, M. L. C.; CASADO, F. C. Análise de perfis longitudinais de drenagens do município de Guarulhos para a detecção de deformações neotectônica. Revista Ung Geociências, Guarulhos, v. 6, n. 8, p. 64-78, 2003.

ARIZA-VILLAVERDE, A. B.; JIMÉNEZ-HORNERO, F. J.; DE RAVÉ, E. GUTIÉRREZ. Influence of DEM resolution on drainage network extraction: a multifractal analysis. Geomorphology, v. 241, p. $243-254,2015$. 
BERA, A. K., SINGH, V.; BANKAR, N.; SALUNKHE, S. S.; SHARMA, J. R. Watershed delineation in flat terrain of Thar Desert Region in North West India-A semi-automated approach using DEM. Journal Of The Indian Society Of Remote Sensing, v. 42, n. 1, p. 187-199. 2014.

CHRISTOFOLETTI, A. Modelagem de sistemas ambientais. 1. ed. São Paulo: Edgard Blücher, 1999. 256p.

FELICÍSIMO, A. M. Modelos digitales de terreno. Introducción y aplicaciones en las Ciencias ambientales (digital elevation model: introduction and applications to environmental sciences). 1. ed. Oviedo: Pentalfa, 1994. 118p.

CPRM, Serviço Geológico Do Brasil. Disponível em: <www.geobank.sa.cprm.gov.br> acesso em: 25 abr. 2018.

DAS, S.; PATEL, P. P.; SENGUPTA, S. Evaluation of different digital elevation models for analyzing drainage morphometric parameters in a mountainous terrain: a case study of the SupinUpper Tons Basin, Indian Himalayas. Springer Plus, v. 5, n. 1, p. 1544-1544, 2016.

FREY, O.; SANTORO, M.; WERNER, C. L.; WEGMÜLLER, U. DEM-Based sar pixel-area estimation for enhanced geocoding refinement and radiometric normalization. IEEE Geoscience And Remote Sensing Letters, v. 10, n. 1, p. 48-52, 2012.

HORTON, R. E. Erosional development of streams and their drainage basins: hrydrophysial approach to quantitative morphology. Geol. Soc. America bulletin, v. 56, n. 3, p. 275-370, 1945.

HSU, Y. C.; PRINSEN, G.; BOUAZIZ, L.; LIN, Y. J.; DAHM, R. An investigation of dem resolution influence on flood inundation simulation. Procedia Engineering, v. 154, p. 826-834, 2016.

MIRANDA, E. E. Brasil em relevo. Campinas: Embrapa Monitoramento Por Satélite, 2005. Disponível em: <http://www.relevobr.cnpm.embrapa.br>. Acesso em: 6 nov. 2017.

MOKARRAM, M.; HOJATI, M. Morphometric analysis of stream as one of resources for agricultural lands irrigation using high spatial resolution of digital elevation model (dem). Computers and Electronics in Agriculture, v. 142 p. 190-2000, 2017.

MS. Mato Grosso Do Sul (Estado). Atlas multirreferencial. Campo Grande: Secretaria de Planejamento e Coordenação Geral (Seplan), 1990. Escala1:1.500.000.

NIKOLAKOPOULOS, K. G.; CHOUSSIAFIS, C.; KARATHANASSI, V. Assessing the quality of dsm from alos optical and radar data for automatic drainage extraction. Earth Science Informatics, v. 8, n. 2, p. 293-307, 2015.

PAN, F.; LIAO, J.; LI, X.; GUO, H. Application of the inundation area-lake level rating curves constructed from the SRTM dem to retrieving lake levels from satellite measured inundation areas.

Computers \& Geosciences, v. 52, p. 168-176, 2013.

PATEL, D. P.; GAJJAR, C. A.; SRIVASTAVA, P. K. Prioritization of Malesari mini-watersheds through morphometric analysis: a Remote Sensing and GIS perspective. Environmental Earth Sciences, v. 69, n. 8, p. 2643-2656, 2013.

PAUL, D.; MANDLA, V. R.; SINGH, T. Quantifying and modeling of stream network using digital elevation models. Ain Shams Engineering Journal, v. 8, n. 3, p. 311-321, 2017. 
SALLUN FILHO, W.; KARMANN, I.; BOGGIANI, P. C.; PETRI, S.; DE SOUZA CRISTALLI, P.; UTIDA, G. A deposição de tufas quaternárias no Estado de Mato Grosso do Sul: proposta de definição da formação serra da Bodoquena. Geologia. USP. Série Científica, v. 9, n. 3, p. 47-60, 2009.

SANTOS, A. M. D.; TARGA, M. D. S.; BATISTA, G. T.; DIAS, N. W. Morphometric analysis of sub-basins Fojo and Perdizes in the city of Campos do Jordão, SP, Brazil. Revista Ambiente \& Água, Taubaté, v. 7, n. 3, p. 195-211, 2012.

SCHUMM, S. A. Evolution of drainage systems and slopes in badlands of Perth Amboy. Geol. Soc. America bulletin, v. 37, p. 597-646, 1956.

SMALL, D. Flattening gamma: radiometric terrain correction for SAR imagery. Ieee Transactions Of Geoscience And Remote Sensing, v. 49, n. 8, p. 3081-3093, 2011.

SOUSA, T. M. I.; PAZ, A. R. How to evaluate the quality of coarse-resolution dem-derived drainage networks. Hydrological Processes, v. 31, p. 3379-3395, 2017.

SREEDEVI, P. D.; SREEKANTH, P. D.; KHAN; H. H.; AHMED, S. Drainage morphometry and its influence on hydrology in an semi arid region: using SRTM data and GIS. Environmental Earth Sciences, v. 70, n. 2, p. 839-848, 2013.

SREEDEVI, P. D.; OWAIS, S.; KHAN, H. H.; AHMED, S. Morphometric analysis of a watershed of South India using SRTM data and GIS. Journal Of The Geological Society Of India, v. 73, n. 4, p. 543-552, 2009.

STRAHLER, A. N. Dimensional analysis applied to fluvial eroded landforms. Geological Society of America Bulletin, v. 69, p. 279-300, 1958

JENSON, S. K.; DOMINGUE, J. O. Extracting topographic structure from digital elevation data for Geographic Information System analysis. Photogrammetric Engineering And Remote Sensing, v. 54, n. 11, p. 1593-1600, 1988.

TONEllO, K. C.; DiAS, H. C. T.; SOUZA, A. L. D.; RIBEIRO, C. A. A. S.; LEITE, F. P. Morphometric characteristics of Cachoeira das Pombas watershed, Guanhães-MG, Brazil. Revista Árvore, Viçosa, v. 30, n. 5, p. 849-857, 2006.

VILLELA, S. M.; MATTOS, A. Hidrologia aplicada. 1. ed. São Paulo: McGraw-Hill do Brasil, 1975. 245p.

WU, S.; LI, J.; HUANG, G. H. A study on DEM-derived primary topographic attributes for hydrologic applications: sensitivity to elevation data resolution. Applied Geography, v. 28, n. 3, p. 210-223, 2008. 